\title{
Projection-Based Needle Segmentation in 3D Ultrasound Images
}

\author{
Mingyue Ding and Aaron Fenster \\ Imaging Research Laboratories, Robarts Research Institute, \\ 100 Perth Drive, London, ON, Canada, N6A 5K8 \\ \{mding, afenster\} @imaging.robarts.ca
}

\begin{abstract}
Our method is composed of three steps: The 3D image is projected along an initial direction, and the needle is segmented in the projected image. Using the projection direction and detected 2D needle direction, a plane containing the needle, called the needle plane, is determined. Secondly, the 3D image is projected in the direction perpendicular to the normal of the needle plane and Step 1 is repeated. If the needle direction in the projected image is horizontal, the needle plane is correct, otherwise Steps $1 \& 2$ are repeated until a correct needle plane has been found. Thirdly, the 3D image is projected along the normal direction of the needle plane. In this projected image, we determine the endpoints of the needle in the projection coordinates. Then, using the relationship between the projection coordinates and the 3D image coordinates, we determine the end points of the needle in the 3D US image. Experiments with agar and turkey phantom 3D US images demonstrated that our method can segment the needle from 3D US images accurately and robustly with a speed of $13 \mathrm{fps}$ on a $1.8 \mathrm{GHz} \mathrm{PC}$ computer.
\end{abstract}

\section{Introduction}

Needles are used in radioactive seed implantation for brachytherapy [1] and sampling a lesion for biopsy. [2,4] In these applications, the 3D position of the needle is required to be obtained accurately and quickly. One of the most promising ways in performing these tasks is to use 3D Ultrasound (US) guidance.

Three-dimensional ultrasound (3D US) is a developing technology that has benefited from advances in computer technology and 3D visualization techniques. $[3,8,9]$. It is now commercially available, $[8,9]$ and is used for a variety of diagnostic applications such as obstetrical, vascular, and urological imaging. [8-10] Using the 3D US technique such as used in Ref. [5], we acquire the 3D US image containing a needle. However, needle segmentation from 3D US images is difficult because of the following reasons: 1. The US image suffers from speckle and shadowing. 2 . Processing of 3D US images involves a large amount of data, e.g., in our experiments the $3 \mathrm{D}$ image is $357 \times 326 \times 352$, i.e., about $40 \mathrm{MB}$. 3. High segmentation accuracy, such as $1^{\circ}$ in orientation and $1 \mathrm{~mm}$ in position, is required in real-time. In order to solve these problems, we developed two 3D needle segmentation methods. [6,7] In Ref. [6], we described an automatic 3D needle segmentation method, which is composed of three steps: First, the 3D image is projected along an initial projection direction, which is perpendicular to the approximate needle orientation, and the needle is segmented in the $2 \mathrm{D}$ projected image. Then, the plane containing the needle 
is determined using the initial projection direction and the needle direction determined in the projected image. Finally a 1D optimization procedure that leads to a minimization of the projected needle area is performed by iteratively choosing the projection direction in the needle plane. However, if the initial projection direction is too far from the actual needle direction, the solution may be trapped in a local minimum and a correct segmentation cannot be ensured. In addition, the insensitivity of the projected needle area to the projection direction also lowers the accuracy of needle segmentation. In order to avoid such problems, we further developed a fast 3D needle segmentation based on using two orthogonal two-dimensional image projections in Ref. [7].

In this paper, we combine the techniques in Refs. [6] and [7] to develop a new 3D needle segmentation technique that is more accurate and robust, especially when a correct 2D needle segmentation in the 2D US images cannot be ensured.
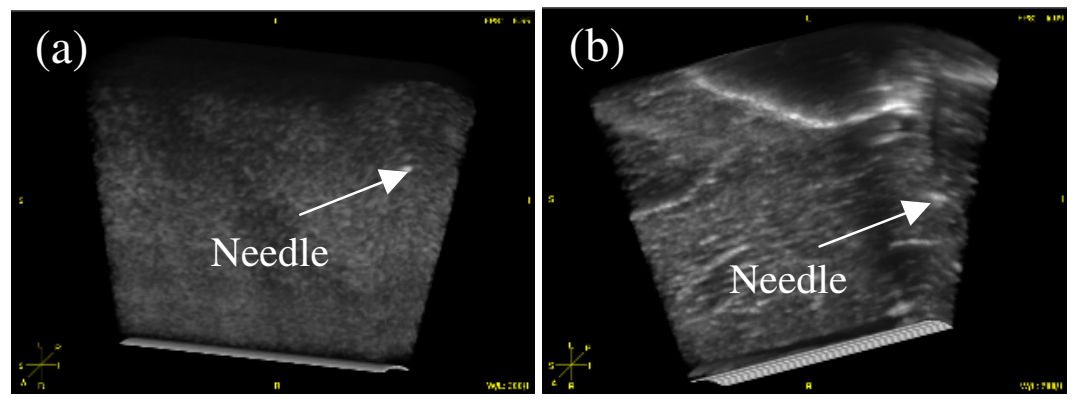

Fig. 1. The multiplanar reformatted view of the 3D US images for the two types of phantoms used in our experiments. (a) Agar phantom. (b) Turkey breast phantom. The arrow is pointing to the endpoint of the inserted needle.

\section{Methods}

\subsection{D US Image Acquisition}

The 3D US images we used in testing our method were all obtained using the Sirus 3D Ultrasound imaging system (Life Imaging Systems, London, Canada) with a fan scanning mechanism. [8-10] This system has been used in both 3D ultrasound guided cryosurgery and brachytherapy, as well as in the development of our 3D ultrasound guided breast biopsy system. [5,11]

The scanning mechanism consists of a mounting device to hold a transducer in place, and a motor system to rotate a side-firing transrectal transducer in contact with the test phantoms used in our experiments. $[12,13]$ The ultrasound machine used in our experiments was an ATL HDI system (Advanced Technology Laboratories, Bothell, Washington) with a side-firing array transrectal transducer. As the transducer was rotated, a video frame grabber digitized the B-mode images at rate of 15 frames/second at $1^{\circ}$ intervals over a $45^{\circ}$ scanning angle. After scanning, the acquired $2 \mathrm{D}$ images were reconstructed into a $3 \mathrm{D}$ image, which was then transferred to our PC computer to test our needle segmentation algorithm. 
To simulate both needle insertions in breast biopsy and prostate therapy, the needle was inserted into the test phantoms approximately parallel to the transducer axis. Two types of phantoms were used in our experiments: agar blocks constructed to provide uniform echogenicity (see Fig. 1(a)), and turkey breasts to provide more complex echogenicity (see Fig. 1(b)). For each phantom, 3D images were obtained with the needle inserted to different depths. We captured 3 images for each phantom. The size of the 3D US images was $357 \times 326 \times 352$ with the voxel dimensions of $0.12 \times 0.12 \times 0.11 \mathrm{~mm}^{3}$. Figure 1 shows the multi-planar reformatted views of one of the agar and turkey breast phantom 3D US images used in the experiments.

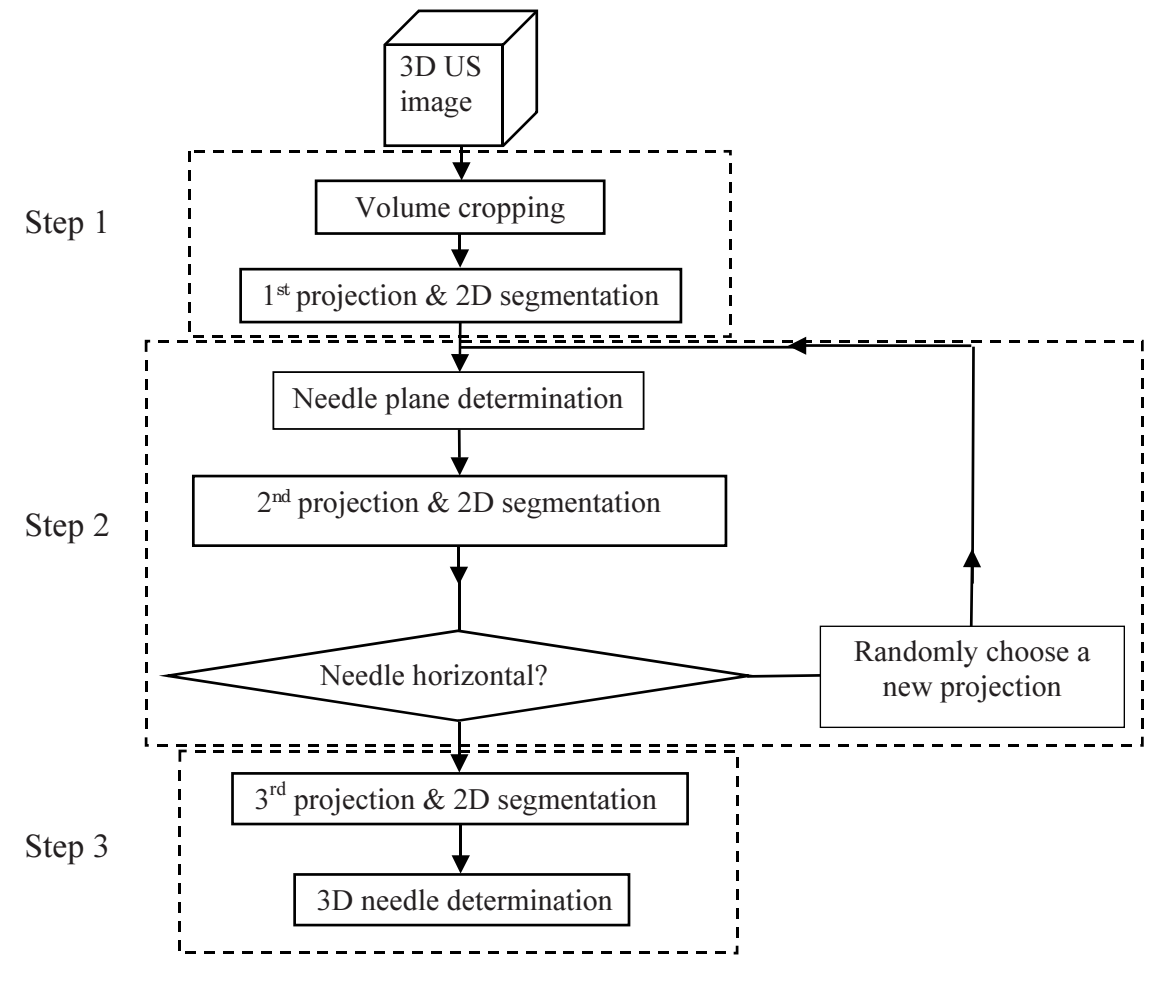

Fig. 2. Diagram of projection-based 3D needle segmentation method.

\subsection{Projection-Based 3D Needle Segmentation Method}

The diagram of our projection-based 3D needle segmentation method is depicted in Fig. 2. It is composed of three steps. First, The 3D US image containing the needle is cropped using a priori knowledge of the approximate needle direction and entrance points. [7] Then, a direction perpendicular to the approximate needle direction is chosen as the initial projection direction. The cropped 3D US image is then volume rendered using ray casting along the initial projection direction with a Gaussian transfer functions described by Eq. (3) in Ref [7] and a projected 2D US image, called the first projected image, is obtained. In the first projected image, we used a $2 \mathrm{D}$ 
needle segmentation technique, such as the flood-filling-based 2D US image segmentation method [7] or the real-time Hough transform technique described in Ref. [14] to segment the needle. Using the initial projection direction and the needle direction segmented from the first projected image, a plane containing the needle, called the needle plane, is determined. In Step 2, we project the cropped 3D image along a direction perpendicular to the normal of the needle plane (see Fig. 3) and perform the 2D needle segmentation in the projected 2D image, called the second projected image. If the $2 \mathrm{D}$ needle segmentation in the $2 \mathrm{D}$ US image is correct, the needle in the second projected image should be horizontal. In this case, we confirm the needle plane determined by Step 1 is correct; otherwise we use the second projection to replace the initial projection and repeat Step 1. This procedure is continued until a correct needle plane is confirmed. In Step 3, we project the 3D image in the third direction parallel to the normal of the needle plane and perform the 2D needle segmentation in the projected 2D image, called the third projected image. From the segmented needle in third projected image, we obtain the coordinates of the endpoints of the needle in the projection coordinate system (see Fig. 3). Using a similar approach as in Section V of Ref. [7], we transform the projection coordinates to the volume coordinates and obtain the final result of the segmented needle in the 3D US image.

\subsection{Evaluation of Needle Segmentation Method}

In order to evaluate the performance of our projection-based needle segmentation method, we must know the actual needle position in the phantom. Because it is difficult to measure the needle in a phantom directly, a physician performed manual needle segmentation for each image and the results were used as the "gold standards" for our evaluation. Our automatic needle segmentation was compared to manual segmentation using the following parameters to measure the segmentation accuracy.

\subsubsection{Angular Deviation $(\Delta \phi)$}

The angular deviation, $\Delta \phi$, was defined by Eq. (1) thus:

$$
\begin{gathered}
\Delta \phi_{i}=\sqrt{\Delta \alpha_{i}^{2}+\Delta \beta_{i}^{2}}, \quad i=1,2, \cdots, 30 \\
\Delta \alpha_{i}=\alpha_{i}-\alpha_{0}, \Delta \beta_{i}=\beta_{i}-\beta_{0}
\end{gathered}
$$

where $\alpha_{0}, \beta_{0}$ are the angles between the $\mathrm{X}$ - and $\mathrm{Y}$-axes and the manually segmented needle, while $\alpha_{i}, \beta_{i}$ are the angles between the $\mathrm{X}$ - and Y-axes and our automatically segmented needle in the $i^{\text {th }}$ segmentation trial. For each 3D US image, we repeated the automatic segmentation 30 times with different initializations of the approximate needle orientation. From Eq. (1), we further defined the average deviation, $\Delta \bar{\phi}$, and standard deviation, $\sigma_{\phi}$, of the needle orientation as:

$$
\overline{\Delta \phi}=\frac{1}{30} \sum_{i=1}^{30} \Delta \phi_{i}, \sigma_{\phi}=\sqrt{\frac{1}{29} \sum_{i=1}^{30}\left(\Delta \phi_{i}-\overline{\Delta \phi}\right)^{2}}
$$




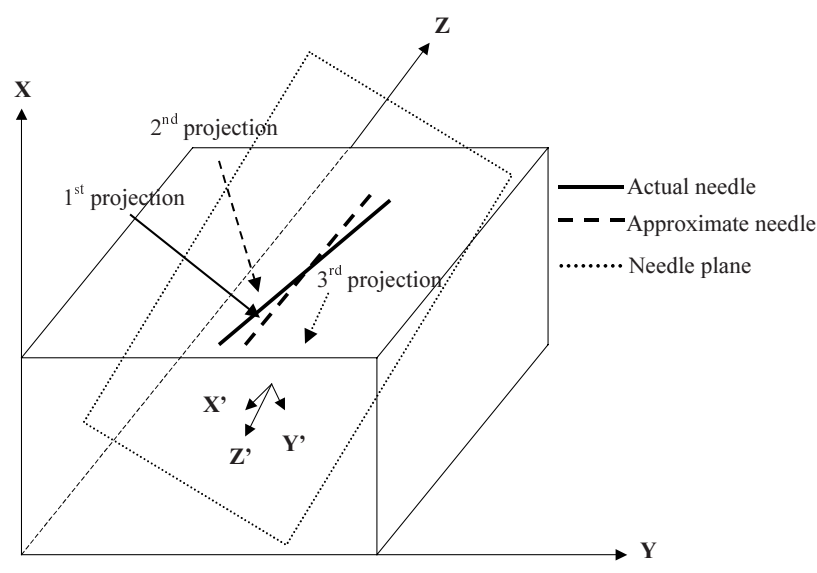

Fig. 3. Configuration of the three projection directions in the volume coordinate system

\subsubsection{Position Deviation $(\Delta p)$}

Suppose the endpoints of the manual segmented needle are $P_{1}=\left(x_{1}, y_{1}, z_{1}\right)$ and $P_{2}=\left(x_{2}, y_{2}, z_{2}\right)$, and their corresponding coordinates obtained from the automatic segmentation in the $i^{t h}$ trial are $P_{i 1}=\left(x_{i 1}, y_{i 1}, z_{i 1}\right)$ and $P_{i 2}=\left(x_{i 2}, y_{i 2}, z_{i 2}\right)$, then we define the position deviation of needle segmentation as $\Delta p_{i}=\max \left\{d\left(P_{i 1}, P_{1}\right), d\left(P_{i 2}, P_{2}\right)\right\}, d\left(P_{1}, P_{2}\right)$ represents the Euclidean distance between the two points $P_{1}$ and $P_{2}$. Similar to the angular deviation, we also define the average and standard deviations in position as described in Eq. (3).

\subsubsection{Segmentation Time}

In order to evaluate the segmentation time more accurately, we measured the computational time for $n$ trials and defined the average segmentation time by $\bar{t}=t / n(t$ is the measured time for $n$ segmentation trials, $n=510$ in our experiments.

\section{Results}

The needle segmentation accuracy results for our method are listed in Table 1. In our experiments, we used agar and turkey breast phantoms (see Fig.1). For each phantom, we inserted a brachytherpy needle into the phantom with different lengths to mimic the needle insertion used in brachytherapy procedure. The inserted needle length, L, varied from $4.0 \mathrm{~mm}$ to $17.6 \mathrm{~mm}$ for agar phantom US images and from $12.5 \mathrm{~mm}$ to $36.7 \mathrm{~mm}$ for the turkey breast phantom US images. The size of the cropped volume we used in the segmentation was $81 \times 81 \times 312$ and the maximal angular deviation between the actual needle and approximate needle, $\Phi$, was $16^{\circ}$. For each 3D US 
image, we segmented the needle 30 times using our method. In each trial, we randomly generated a needle orientation, $\phi$, which was less than or equal to the approximate needle orientation, $\Phi$. In Table $1, \overline{\Delta \phi}$ and $\sigma_{\phi}$ are the average and standard deviations of the angular difference between the manually and automatically segmented needles defined by Eqs. (4) and (5), while $\overline{\Delta p}, \sigma_{p}$ are the average and standard deviations in position. The first 3 rows in Table 1 are the segmentation results of three Agar phantom 3D US images and the $4^{\text {th }}$ row is their average result. Similarly, the segmentation results of the 3 turkey breast images are listed in rows 5 to 8 . From Table 1, it is apparent that the segmentation position deviation using our method varied from $0.44 \mathrm{~mm}$ to $0.60 \mathrm{~mm}$ for the agar images with an average of 0.53 $\mathrm{mm}$ while for the turkey breast phantom images the position error varied from 0.34 $\mathrm{mm}$ to $1.18 \mathrm{~mm}$ with an average of $0.69 \mathrm{~mm}$. The segmentation angular deviation using our method varied from $0.97^{\circ}$ to $1.56^{\circ}$ for the agar images with an average of $1.19^{\circ}$ while for the turkey breast phantom images it varied from $0.70^{\circ}$ to $1.08^{\circ}$ with an average of $0.77^{\circ}$. The standard deviations of the position and angular deviations for both the agar and the turkey breast phantoms are very small (less than $0.033 \mathrm{~mm}$ for the position error and $0.072^{\circ}$ for the angular deviation).

The average computational time given in Table 1 was measured on our Pentium IV $1.8 \mathrm{GHz}$ PC computer. It varied from $70.3 \mathrm{~ms}$ to $76.9 \mathrm{~ms}$, or about $13 \mathrm{fps}$, a nearly real-time performance.

Figure 4 shows the comparison plots of the average segmentation errors versus the value of the maximum angular deviation, $\Phi$, for both agar and turkey breast phantom images, where we varied the value of $\Phi$ from $0^{\circ}$ to $32^{\circ}$ in step of $2^{\circ}$. Figures 4(a) and (b) show the angular average deviations, while Fig. 4(c) and (d) show their average position deviations. For both agar and turkey breast phantoms, we averaged the segmentation errors over the 3 different US images. Figure 4 shows that for the agar phantom US images, the average angular deviation varied from $1.05^{\circ}$ to $1.29^{\circ}$ and the average position error varied from $0.43 \mathrm{~mm}$ to $0.74 \mathrm{~mm}$ for both projection based segmentation approach and the approach described in Ref. [7]. For the turkey breast phantom US images, the segmentation errors using in the projectionbased segmentation approach varied from $0.62^{\circ}$ to $0.82^{\circ}$ in angular deviation and from $0.61 \mathrm{~mm}$ to $0.98 \mathrm{~mm}$ in position, while for the approach described in Ref. [7], the errors varied from $1.80^{\circ}$ to $1.97^{\circ}$ in the angular deviation and from $1.35 \mathrm{~mm}$ to $1.55 \mathrm{~mm}$ in position respectively.

\section{Conclusion}

In this paper, we described a 3D needle segmentation method based on validation of the correct needle plane. The experiments with Agar and turkey breast phantom US images demonstrated that our method could perform accurate needle segmentation from 3D US images in nearly real-time on a PC computer. This approach is being 
used in our Robot-based interventional biopsy and therapy system and will be further evaluated in cryosurgery and biopsy. Specifically, our experiments demonstrated the following conclusions:

1) For the US images with complex echogenicity, such as the Turkey breast phantom image in Fig. 1(b), our projection-based approach segments the needle more accurately than the approach described in Ref. [7].

2) The segmentation errors are depended on the maximum angular deviation, $\Phi$. Usually, the greater the value of $\Phi$, the greater the position error and the angular error will be.

3) The position of the segmented needle is more sensitive than the orientation of the needle to the variation of the maximum angular deviation, $\Phi$. Fortunately, its effects on the position and angular error are small if the value of $\Phi$ is less than $32^{\circ}$, which is the usual case in brachytherapy as the approximate position and orientation of the inserted needles are known.
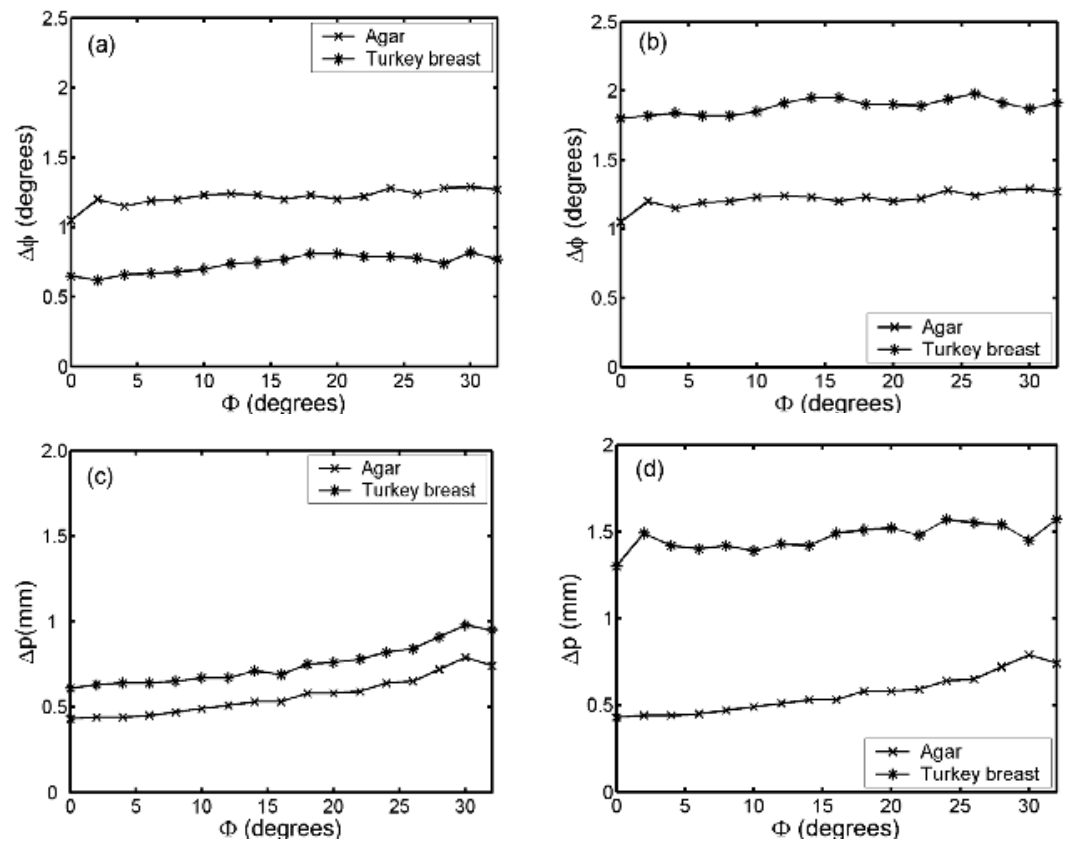

Fig. 4. The segmentation error comparison between our 3D needle segmentation method and the approach described in Ref. [7]. Here, $\Phi$ is the maximum angular deviation between the actual needle and approximate needle. $\overline{\Delta \phi}, \sigma_{\phi}$ are the average and standard deviation of the angular error, while $\overline{\Delta p}, \sigma_{p}$ are the average and standard deviation of the position error. (a) and (c) are respectively the angular and position errors of the needle segmentation using our projection based approach, while (b) and (d) are the results obtained using the approach described in Ref. [7]. 
Table 1. The segmentation error for both agar and turkey breast phantom images using our segmentation method. $\overline{\Delta \phi}, \sigma_{\phi}$ are the average and standard deviation in orientation, while $\overline{\Delta p}, \sigma_{p}$ are the average and standard deviation in position. $\mathrm{L}$ is the inserted length of the needle in the phantom.

\begin{tabular}{lcccccc}
\hline Image & $\overline{\Delta \phi}\left({ }^{\circ}\right)$ & $\sigma_{\phi}\left({ }^{\circ}\right)$ & $\Delta p(\mathrm{~mm})$ & $\sigma_{p}(\mathrm{~mm})$ & $\bar{t}(\mathrm{~ms})$ & $\mathrm{L}(\mathrm{mm})$ \\
\hline \hline Agar 1 & 1.56 & 0.006 & 0.55 & 0.033 & 70.3 & 4.0 \\
Agar 2 & 0.97 & 0.017 & 0.44 & 0.001 & 72.5 & 16.8 \\
Agar 3 & 1.04 & 0.003 & 0.60 & 0.012 & 74.1 & 17.6 \\
\hline Average & 1.19 & 0.008 & 0.53 & 0.015 & 72.3 & 12.8 \\
\hline Turkey 1 & 1.08 & 0.072 & 0.34 & 0.010 & 73.4 & 12.5 \\
Turkey 2 & 0.70 & 0.017 & 0.56 & 0.013 & 75.2 & 26.3 \\
Tukey 3 & 0.52 & 0.020 & 1.18 & 0.018 & 76.9 & 36.7 \\
\hline Average & 0.77 & 0.036 & 0.69 & 0.014 & 75.2 & 25.2 \\
\hline
\end{tabular}

\section{References}

1. Nag S.: Principles and practice of brachytherapy. Futura Publishing Company, Armark, New York (1997)

2. Fornage BDL: Sonographically guided needle biopsy of nonpalpable breast lesions. Journal of Clinic Ultrasound, Vol. 27 (1999) 385-398

3. Peters T. M.: Image-guided surgery: From X-rays to Virtual reality. Computer Methods in Biomedical Engineering, Vol. 4 (2000) 27-57

4. Liberman L., Feng T. L., Dershaw D. D., Morris E. A., Abramson A. F.: US-guided core breast biopsy: Use and cost-effectiveness. Radiology, Vol. 208 (1998) 717-723

5. Chin J. L., Downey D. B., Onik G., Fenster A.: 3-dimensional Ultrasound Imaging and its Application to Cryosurgery for Prostate Cancer. Techniques in Urology, Vol. 2, No. 4 (1997) 187

6. Ding M., Guan W., Cardinal H.N., Fenster A.: Automatic Needle Segmentation from 3D Ultrasound Images. SPIE on Medical Imaging, Vol. 4681, February 22-24, 2002, San Diego, CA, USA, 65-76

7. Ding M., Cardinal N., Fenster A.: Automatic Needle Segmentation in 3D Ultrasound Images using Orthogonal 2D Image Projections. Medical Physics, Vol. 30, No. 2 (2003) 222-234,.

8. Nelson T.R., Downey D.B., Pretorius D.H., Fenster A.: Three-Dimensional Ultrasound. Lippincott, Williams, and Wilkins, Philadelphia. (1999) 11-29

9. Fenster A., Downey D., Cardinal N.: Topical review: 3-dimensional Ultrasound Imaging. Physics in Medicine and Biology, Vol. 46 (2001) R67-R99

10. Fenster A., Downey D.: 3-Dimensional Ultrasound Imaging: A Review. IEEE Engineering in Med. and Biol., Vol. 15 (1996) 41-51

11. Smith W., Surry K., Mills G., Downey D., Fenster A.: Three-Dimensional UltrasoundGuided Core Needle Breast Biopsy. Ultrasound in Medicine and biology, Vol. 27, No. 8 (2001) 10425-1034 
12. Tong, S., Downey, D. B., Cardinal, H. N., Fenster, A.: Three-dimensional Ultrasound Prostate Imaging System. Ultrasound in Medicine and Biology, Vol. 22, No. 6 (1996) 735-746

13. Elliot T., Downey D. B., Tong S., McLean C. A., Fenster A.: Accuracy of Prostate Volume Measurements In Vitro Using Three-Dimensional Ultrasound. Academic Radiology, Vol. 3, No.5 (1996) 401-406

14. Ding M., Fenster A.: A real-time biopsy needle segmentation technique using Hough transform. Medical Physics, Vol. 30, No. 8 (2003) 